\title{
An update on aureochromes: Phylogeny - mechanism - function
}

\author{
Peter G. Kroth ${ }^{\mathrm{a}, *}$, Christian Wilhelm ${ }^{\mathrm{b}}$, Tilman Kottke ${ }^{\mathrm{c}}$ \\ a Department of Biology, University of Konstanz, 78457 Konstanz, Germany \\ b Institute of Biology, University of Leipzig, Johannisallee 21-23, 04103 Leipzig, Germany \\ c Department of Chemistry, Physical and Biophysical Chemistry, Bielefeld University, Universitätsstraße 25, 33615 Bielefeld, Germany
}

\author{
Keywords: \\ Aureochrome \\ bZIP \\ Diatom \\ LOV \\ Structure \\ Transcription factor
}

\begin{abstract}
A B S T R A C T
Light is important for algae, as it warrants metabolic independence via photosynthesis. In addition to the absorption of light by the photosystems, algae possess a variety of specific photoreceptors that allow the quantification of the light fluxes as well as the assessment of light qualities. About a decade ago, aureochromes have been described in the xanthophyte alga Vaucheria frigida. These proteins represent a new type of blue light photoreceptor as they possess both a light-oxygen-voltage (LOV) domain for light reception as well as a basic region leucine zipper (bZIP) domain for DNA binding, indicating that they represent light-driven transcription factors. Aureochromes so far have been detected only in a single group of algae, photosynthetic stramenopiles, but not in any other prokaryotic or eukaryotic organisms. Recent biophysical work on aureochromes in the absence and the presence of DNA revealed the mechanism of allosteric communication between the sensor and effector domains despite their unusual inversed arrangement. Different molecular models have been proposed to describe the effect of light on DNA binding. Functional characterization of mutants of the diatom Phaeodactylum tricornutum, in which the aureochrome genes have been silenced or deleted, indicate that different aureochromes may have different functions, being involved in central processes like light acclimation and regulation of the cell cycle.
\end{abstract}

\section{Introduction}

Light for photoautotrophic organisms not only is the driving factor for the energy-providing process of photosynthesis, it also represents an important environmental signal, triggering various physiological responses. Cells and organisms are capable of evaluating the intensity as well as the quality of the light (Kianianmomeni and Hallmann, 2014). For this purpose, they utilize so-called photoreceptors, these are proteins that are able to absorb specific wavelengths in the visible and ultraviolet range of light (Hegemann, 2008). Light absorption by these proteins results in conformational changes that may transduce this information in potentially multi-step signaling processes to the respective cellular regulatory system (Duanmu et al., 2017). While sunlight is composed of a wide spectrum of wavelengths from ultraviolet to infrared, most of the photoreceptors in plants and algae absorb either in the UV/blue or in the red/far-red spectral range of wavelengths (Depauw et al., 2012). Phytochromes, to current knowledge, are the only widely distributed photoreceptors that are absorbing red light. These proteins possess a linear tetrapyrrole as a prosthetic group and can be switched reversibly from an active to an inactive state by red and far-red light, respectively (Chen and Chory, 2011). Recently, spectrally tuned phytochromes have been identified in algae that cover the whole visible spectral region (Rockwell et al., 2014), possibly as adaptation to the light conditions in the ocean. In contrast, a larger number of different blue light photoreceptors are known, most of which are using a flavin as chromophore. Cryptochromes are binding flavin adenine dinucleotides (FAD). They are blue/UV-A light receptors that form a large and diverse family with photolyases that are involved in repair of UVdamaged DNA (Chaves et al., 2011; Mittag et al., 2017; König et al., 2017; Kottke et al., 2017). Another group of blue light receptors contain light-oxygen-and-voltage (LOV) domains (Crosson et al., 2003), which are binding a flavin mononucleotide (FMN). Prominent representatives here are the phototropins and the aureochromes. In euglenophyta, there are also other blue light sensors using FAD (BLUF) (Kennis and Mathes, 2013). Finally, microbial rhodopsins have been identified to be involved in light-induced locomotion of green flagellates (Foster et al., 1984; Ernst et al., 2014). In Chlamydomonas reinhardtii, these lightgated ion channels named channelrhodopsins are located in the

\footnotetext{
Corresponding author.

E-mail address: Peter.Kroth@uni-konstanz.de (P.G. Kroth).
} 
photosensory eyespot (Suzuki et al., 2003).

In algae, a number of physiological responses to blue light are known. One early studied example is phototaxis (Halldal, 1958), the ability of motile algae to move either to or away from light. In the filamentous green alga Mougeotia scalaris, photoorientation of the ribbon-shaped chloroplast has been demonstrated to be induced by blue light, probably by phototropins (Gabryś et al., 1984; Suetsugu et al., 2005). For this alga, as well for mosses and ferns (Li et al., 2014), a new type of photoreceptor has been described recently, neochrome, which essentially is a fusion protein of a phytochrome and a phototropin. The role of this chimeric photoreceptor is unknown, especially as the neochrome LOV domains apparently cannot bind flavins (Suetsugu et al., 2005). Also in diatoms, blue light-stimulated chloroplast movement and aggregation has been demonstrated (Shihira-Ishikawa et al., 2007).

In this review, we focus on the distribution, the structural response, as well as the functional characterization of aureochromes, light driven transcription factors that just recently have been discovered (Takahashi et al., 2007) in some stramenopiles, including brown algae (Ishikawa et al., 2009), diatoms (Bowler et al., 2008), raphidophytes (Jia et al., 2017), and eustigmatophytes (Vieler et al., 2012).

\section{Distribution of aureochromes in algae}

Aureochromes have been originally identified in the xanthophyte alga Vaucheria frigida in 2007 (Takahashi et al., 2007) and have been termed aureochromes referring to "aurum" (latin for gold), because of the golden-yellow color of some stramenopiles. Vaucheria is a siphonous alga, which means that it consists of giant coenocytic unicells that are not divided by separating cell walls. There had been very early reports that Vaucheria responds to blue light with phototropism (Kataoka, 1979), side branch formation (Takahashi et al., 2001), and plastid movement (Blatt and Briggs, 1980). Takahashi et al. (2007) screened genomic Vaucheria sequences for LOV domains, which are common domains involved in different sensory processes in pro- and in eukaryotes (Herrou and Crosson, 2011). The authors then identified so far unknown genes for potential photoreceptors with an unusual domain combination, possessing a LOV domain at the C-terminus, as well as a basic-region leucine zipper (bZIP) domain, indicating that these proteins might also have DNA-binding capacities and may act as transcription factors. In $V$. frigida, two orthologs were identified, $V f A U R E O 1$ and VfAUREO2. Using an RNAi approach to silence these two genes individually, Takahashi et al. (2007) could demonstrate that both VfAUREOs are involved in photomorphic responses of Vaucheria, and that VfAUREO2 specifically acts as a negative regulator for the differentiation of sex organs. Silencing of both VfAUREO1 and VfAUREO2 simultaneously induced abnormal tube morphology. Interestingly, blue light-induced chloroplast movement was not affected in these mutants, indicating the presence of further blue light receptors. With the upcoming availability of genomic DNA sequences, researchers tried to identify aureochromes in other algae either by sequence similarity (Deng et al., 2014; Schellenberger Costa et al., 2013) or via PCR amplification (Ishikawa et al., 2009). Interestingly, although proteins containing LOV domains are widely distributed in all kinds of organisms (Glantz et al., 2016), aureochromes so far only have been detected in an algal group named stramenopiles (Ishikawa et al., 2009; Schellenberger Costa et al., 2013), with the exception of the related, but generally non-photosynthetic, oomycetes (Fig. 1). Aureochromes apparently are also completely missing in red algae, which are considered to represent the endosymbiotic ancestors of stramenopile plastids (Archibald, 2015), as well as in related algal groups like cryptophytes or haptophytes. Finally, there are also no indications that aureochromes might be present in green algae and in land plants (Fig. 1). This suggests that either the so far unknown host cell of secondary endosymbiosis may have provided the aureochrome gene, or that the aureochromes, possessing this unique combination of LOV and bZIP domains, evolved very early within the stramenopiles, possibly by domain shuffling (Di
Roberto and Peisajovich, 2014). A close relative of aureochromes, the blue light receptor phototropin, contains two LOV domains and a Ser/ Thr kinase domain for signal transduction (Christie, 2007; Li et al., 2015), but no DNA binding domain. Phototropins have only been detected so far within the Viridiplantae, thus in green algae and land plants (Li et al., 2015), but are missing in red algae. This makes it unlikely that aureochromes might have evolved from phototropins directly via domain shuffling. This notion is supported by phylogenetic studies by Ishikawa et al. (2009), demonstrating that the aureochrome LOV domains are phylogenetically separated from both LOV1 and LOV2 domains of phototropins.

\section{Classification of aureochromes}

Because of their apparently different functions, the Vaucheria aureochromes have been termed VfAUREO1 and VfAUREO2 (Takahashi et al., 2007). After the identification of aureochromes in other organisms, a similar strategy first has been pursued by simply numbering the genes/ proteins, even if there were more than two aureochrome genes present in a species. A closer look at the Vaucheria aureochromes, however, reveals that the two VfAUREOs are structurally different. VfAUREO2 shows a mutation, which prevents binding of an essential flavin, and which abolishes light absorbance in the blue range. Structural analyses of PtAUREO2 indicate that a steric hindrance by a methionine residue within its binding cavity is responsible here (Banerjee et al., 2016a). Therefore, both VfAUREO2 and PtAUREO2, strictly speaking, are not photoreceptors, but transcriptions factor that may interact with AUREO1 proteins by forming DNA binding heterodimers. Genetic modification of PtAUREO2 from the diatom Phaeodactylum tricornutum allowed the restoration of flavin binding (Serif et al., unpublished). Introducing, in a reciprocal experiment, a point mutation at the same site into PtAUREO1a results in a similar loss of flavin binding (Banerjee et al., 2016a). When analyzing the four aureochromes from $P$. tricornutum and from other algae, Schellenberger Costa et al. (2012) observed that all studied groups possessed an AUREO2 protein. The other three PtAUREOs are functionally and phylogenetically more related to VfAUREO1, and thus have been named PtAUREO1a/1b/1c, accordingly. However, phylogenetic analysis indicate three different branches of AUREO1 proteins (Schellenberger Costa et al., 2013). This separation is supported by the gene expression patterns of the four $P$. tricornutum aureochromes. The differential expression of PtAUREO1a/1a/1c/2 was studied in light- and circadian-dependent transcript analyses with cells grown in a day/night cycle or kept in the dark for at least one day (Banerjee et al., 2016b). The results show individual transcription patterns for the different aureochromes. While the non-flavin-binding PtAUREO2 appears to be expressed in a time- and light-independent manner, lacking any significant light/circadian regulation, all other AUREO homologs showed differential expression patterns throughout the day. PtAUREO1a revealed a light-independent circadian regulation, being upregulated during the day both in day/night cycles as well as in continuous darkness. PtAUREO1b transcripts instead were significantly increased in illuminated cultures compared to the cultures grown in darkness, while again PtAUREO1c was strongly expressed during the day in both conditions (Banerjee et al., 2016b). These findings indicate the presence of different amounts of the individual aureochromes in P. tricornutum cells in the diel cycle, suggesting either distinctly regulatory, or temporal functions. Considering that aureochromes potentially may form heterodimers, the combination of dominant aureochrome species in a cell at a certain time point may define their regulatory properties by their specific DNA binding affinities.

\section{Mechanism of aureochromes}

\subsection{Differences to other LOV-based photoreceptors}

Aureochromes and phototropins share the same light-absorbing unit, the LOV domain. Blue light absorption causes an adduct formation 


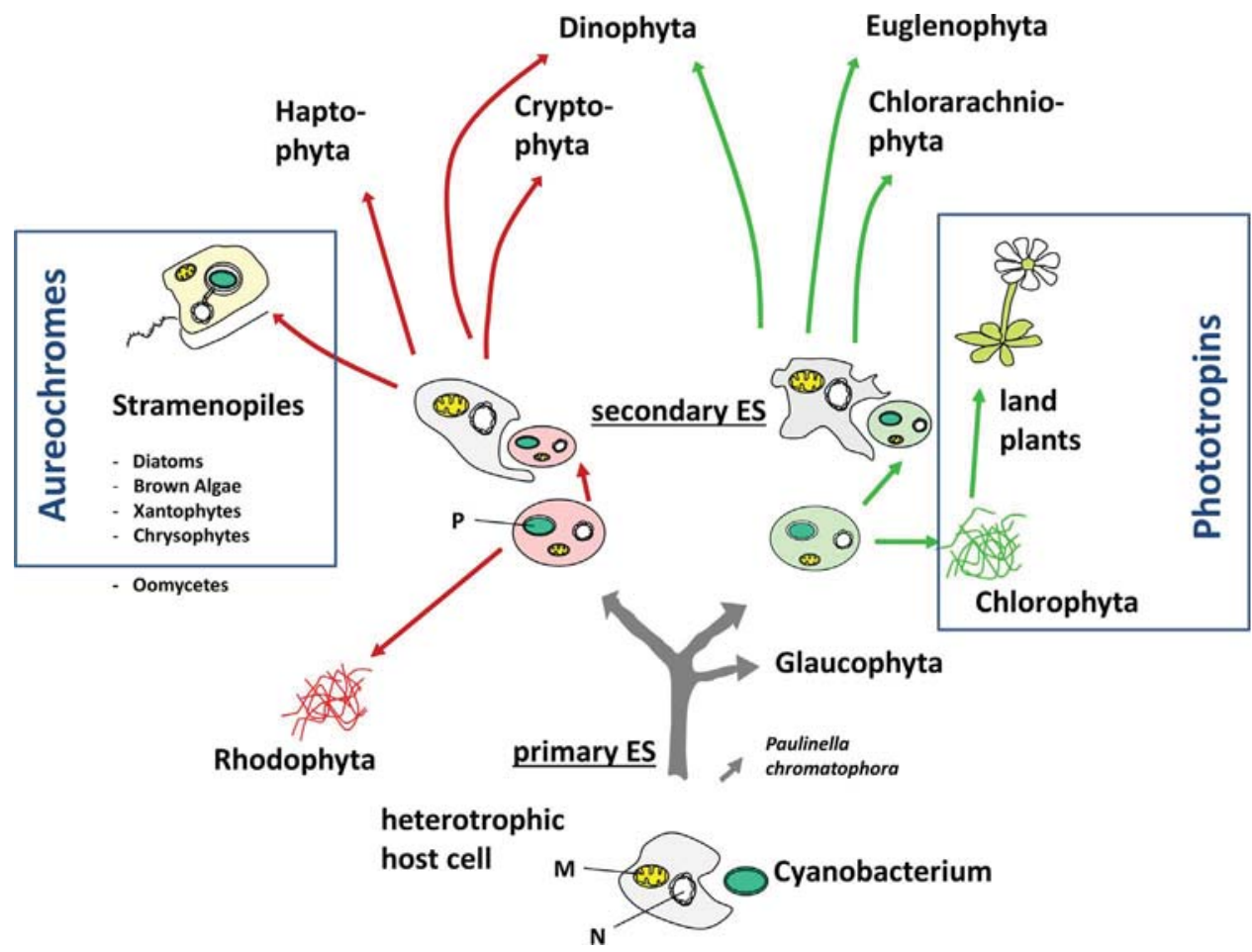

Fig. 1. Occurrence of aureochromes and phototropins in different algal groups. Simplified schematic scenario of major endosymbiotic events in plastid evolution and the respective algal groups. Primary plastids originated via the endosymbiotic integration of a cyanobacterium into a heterotrophic host cell (bottom of the figure), resulting in plastids with two surrounding membranes in glaucophytes, red algae, green algae, and their close relatives, the land plants. Both, ancestors of modern red algae and green algae, served as endosymbionts for secondary endosymbioses in which eukaryotic host cells took up the respective eukaryotic algae. ES: Endosymbiosis, P: Plastid; M: Mitochondrium; N: Nucleus. Scheme based on Kroth (2015).

A
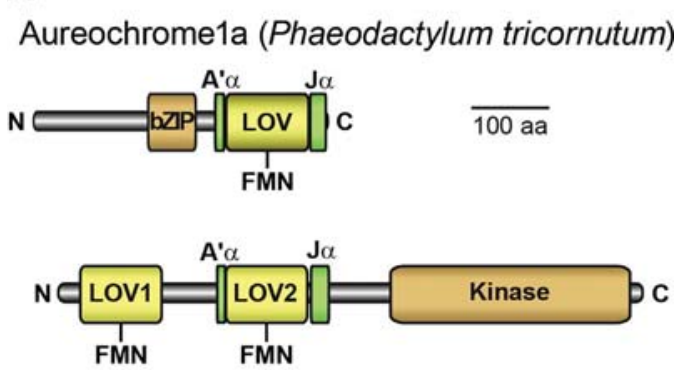

Phototropin (Chlamydomonas reinhardtii)

B

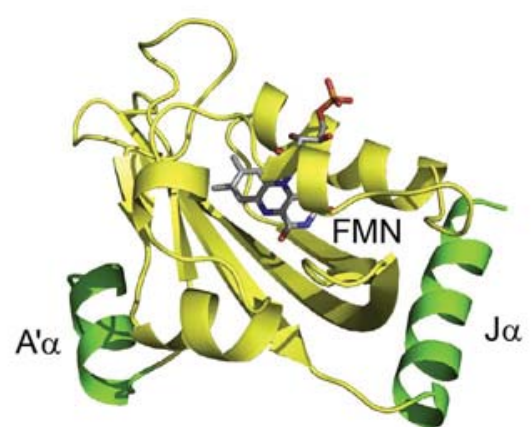

Fig. 2. (A) Domain topology of aureochrome as compared to phototropin. The sequence of the LOV sensor domains and the respective effector domains is inversed in aureochromes. (B) Crystal structure of the LOV monomer of AUREO1a from Phaeodactylum tricornutum (PDB 5A8B). The positions of the chromophore flavin mononucleotide (FMN) and the flanking helices $\mathrm{A}^{\prime} \alpha$ and $\mathrm{J}^{\prime} \alpha$ are depicted.

of flavin and a nearby cysteine within a few microseconds in both photoreceptors starting the signaling cascade (Kerruth et al., 2014; Swartz et al., 2001; Toyooka et al., 2011). However, two key differences are evident in their domain topology. Firstly, the effector domain of aureochromes is a bZIP domain instead of a kinase. bZIP domains comprise a common DNA-binding motif of eukaryotes, which has not been identified before in other members of the LOV protein family, suggesting the function of aureochromes as light-regulated transcription factors (Takahashi et al., 2007). Light-dependent regulation of DNA binding by LOV has been demonstrated for the bacterial EL222 previously, which contains a helix-turn-helix as another common motif instead (Nash et al., 2011). Secondly, the domain topology is inverted as compared to all other characterized LOV proteins, with the sensory domain being located at the C-terminus of the receptor (Fig. 2). Although such a domain inversion has been identified by genetic screens also for other LOV proteins (Losi and Gärtner, 2008), it can be found only in a very small group of all related sequences (Losi, 2004; Glantz et al., 2016). The inverted arrangement raised the general question of whether a communication exists between the LOV and the bZIP domains that may influence DNA binding. Alternatively, a different effector might bind in vivo to the LOV domain.

\subsection{Communication between sensor and effector domain}

In phototropin, the communication between LOV and kinase domains is mediated by the linking $\mathrm{J} \alpha$ helix, which is C-terminally flanking the LOV domain. This Ja helix unfolds upon illumination of LOV (Harper et al., 2003) and thereby releases the inhibition of the kinase by LOV to enable autophosphorylation (Harper et al., 2004; Matsuoka and Tokutomi, 2005). Surprisingly, in aureochrome-LOV this $\mathrm{J} \alpha$ helix plays a decisive role as well despite being located at the Cterminus of the receptor. Unlike in phototropin, the $\mathrm{J} \alpha$ regulates allosterically the fold of the N-terminally flanking A' $\alpha$ helix (Herman and Kottke, 2015). The subsequent unfolding of the A' $\alpha$ helix then exposes a high affinity dimerization site and enables the formation of the light state dimer of LOV (Heintz and Schlichting, 2016; Herman and Kottke, 2015; Herman et al., 2013). Even in the full-length aureochrome, the Ja helix plays a crucial role in the formation of the light state of the receptor (Banerjee et al., 2016b). The bZIP domain responds to the activation of the LOV domain and to the unfolding of J $\alpha$ and A' $\alpha$ by a loss in the helical secondary structure (Banerjee et al., 2016b). This process most likely proceeds with the time constant of $160 \mathrm{~ms}$ that has been assigned to a conformational change in the bZIP domain (Toyooka et al., 2011). These findings provide evidence for a direct 
communication between the two domains. In the presence of DNA, the helical fold of the bZIP domain is increased by light (Banerjee et al., 2016b), leading to a rigidification of the domain (Heintz and Schlichting, 2016). Some results even point to an additional backward allosteric communication, through which DNA binding to bZIP influences the kinetics of the LOV domain recovery (Banerjee et al., 2016b).

\subsection{The unclear oligomerization state in the dark and the impacts of light and redox potential}

The oligomerization state of full-length aureochromes and its dependence on light is under debate. In the dark, PtAUREO1a has been observed as a monomer in equilibrium with a dimer (Heintz and Schlichting, 2016) or as a dimer/higher oligomer (Banerjee et al., 2016b), the latter being in agreement with the general tendency of bZIP domains to dimerize. It is unclear at present, whether the LOV domains within the full-length receptor also form a dimer, because conflicting results have been obtained (Banerjee et al., 2016b; Heintz and Schlichting, 2016). For VfAUREO1, a monomeric form of the full-length receptor has been found to be present under reducing conditions and at low concentrations in the dark (Hisatomi et al., 2014). The light-induced dimerization of LOV then leads to association of the monomers, which is the rate-limiting step in the process of DNA binding (Akiyama et al., 2016). The comparison is further complicated by the fact that VfAUREO1, but not its homologues from $P$. tricornutum, contains solvent-accessible cysteines, which cause a covalent dimerization under oxidizing conditions. Furthermore, the weakly conserved N-terminal extension of aureochromes also affects the oligomerization state (Banerjee et al., 2016b). Based on the conflicting data on the oligomerization state of the dark form of aureochromes, very different effects of light on the oligomerization equilibrium have been found and discussed (Banerjee et al., 2016b; Heintz and Schlichting, 2016; Hisatomi et al., 2014; Toyooka et al., 2011). It should be noted in this context that only the dimeric bZIP domain is capable of binding DNA, a fact which directly links the oligomerization state to the binding affinity.

\subsection{Current models for signaling induced by light}

Up to now, four models have been put forward, and have been supported by experiments, to explain the effect of light on the interaction of aureochromes with DNA. For a monomeric aureochrome, it is conceivable that light-induced dimerization directly regulates DNA binding (Hisatomi et al., 2014). If the aureochrome is in the dimeric state already in the dark, light-induced dimerization of LOV may lead to an increase in distance of the bZIP domains and thereby expose sites important for DNA interaction (Toyooka et al., 2011), which can be summarized as a 'see saw' mechanism. In contrast, the LOV domain monomers may bind directly to the leucine zipper of the bZIP domains in the dark (Heintz and Schlichting, 2016). This latter model is supported by the fact that the linker region between LOV and bZIP is not conserved and seems to be unstructured. Then, dimerization of the LOV domains by light may result in an elongation of the dimer and an allosteric increase in affinity to DNA (Heintz and Schlichting, 2016). Finally, the rotation of the LOV domains in the progression from dark to light dimer may lead to a rigidification of bZIP without changes in the overall structural envelope (Banerjee et al., 2016b). The DNA binding might then be regulated on a more subtle level, in which light enhances the specificity of DNA binding and causes a tilt in the DNA without a direct effect on the affinity (Banerjee et al., 2016b).

While many important steps in the aureochrome mechanism have been revealed recently, further investigations are necessary to finally clarify how the DNA binding is modified by light. It would be instrumental to resolve a crystal structure of the bZIP-LOV module, thereby greatly extending our current knowledge which is limited to the structures of the aureochrome-LOV domain in the dark and light states (Banerjee et al., 2016a; Heintz and Schlichting, 2016; Mitra et al.,
2012). Furthermore, it should be noted that the role of the N-terminal extension is unclear, which necessitates more parallel studies on the full-length receptor and the bZIP-LOV module. Finally, another rewarding focus of future research would be to reveal the biophysical differences between AUREO1a, AUREO1b and AUREO1c to complement the respective physiological studies.

\section{The physiological function of aureochromes in diatoms}

\subsection{The knowledge about the physiological functions of photoreceptors in} diatoms is very scarce

Although about a decade has passed since the discovery of aureochrome in $V$. frigida, their function in unicellular algae, especially in diatoms, is still enigmatic. For several reasons, the characterisation of aureochromes in diatoms is challenging. The control of photomorphogenesis, as observed in Vaucheria, usually is not a useful marker in unicellular cells. Therefore, physiological responses to environmental cues might represent a much better accessible phenotype for cells with altered aureochrome contents. Ishikawa et al. (2012), for instance, observed that cell size and motility is blue-light (BL)-sensitive in the chrom-alveolate alga Ochromonas danica, but they also stated that this observation cannot be solely attributed to the function of aureochromes. Therefore, simple BL on/off experiments appear not to be promising for aureochrome characterization, considering that diatoms contain a large number of additional $\mathrm{BL}$ receptors belonging to the group of cryptochromes (see König et al., 2017), which may interfere with the aureochromes. For $C$. reinhardtii, it has been shown that different BL receptors may form a regulatory network (see Mittag et al., 2017), therefore, at present, the best strategies to identify specific aureochrome functions include the generation of aureochrome-specific mutants, or the identification of specific interaction partners of aureochromes. Here, Huysman et al. (2013) could show that the diatomspecific Cyclin 2 ( $d s C Y C 2$ ) plays a central role in the cell cycle control in diatoms after the onset of light. They also demonstrated that dsCYC2 is specifically targeted by PtAUREO1a, linking BL sensing to cell cycle progression.

Understanding the mode of action of BL receptors when controlling central metabolic processes is further complicated by the fact that BL not only is a signal from the environment, but also a part of the incident light, which is absorbed as energy source for photosynthesis. Therefore, $\mathrm{BL}$, as an environmental signal, may interfere with light acclimation reactions. Accordingly, an experimental design is needed which is able to differentiate between photosynthetic light acclimation reactions and specific BL controlled physiological reactions. Many studies in the past used the same amount of incident irradiance to test light quality effects. Such an experimental design, however, can be misleading, because the cells absorb different wavelengths with a different efficiency. This means that the same incident photon flux may lead to a different absorbed photon flux per cell, which may strongly affect physiological responses. Therefore, when performing BL or red light (RL) studies, it is essential to choose experimental conditions, which guarantee that the number of absorbed photons is identical under both light qualities (for details see Schellenberger Costa et al., 2013). This is even more important for diatoms, because recent studies have shown that light acclimation reactions in these algae differ significantly from those of green algae and higher plants (Wilhelm et al., 2014). In green algae, the functional analysis of STN7 mutants from Chlamydomonas (STNs are protein kinases that are involved in antenna phosphorylation), has demonstrated the intrinsic interaction of light acclimation and the ability to adjust the energy distribution between the photosystems by state transitions. These state transitions are under control of the redox state of the plastoquinone pool, which is oxidized in the dark and becomes reduced in the light (Tikkanen et al., 2012). In diatoms, it was shown that wavelength dependent state transitions are lacking, because both photosystems are functionally coupled to specific fucoxanthin- 
binding complexes (FCPs) that show identical absorption spectra, independent of whether they deliver their excitonic energy to PSII or to PSI (Lepetit et al., 2007). In addition, the PQ pool in diatoms can remain in a reduced state, even in the dark, for longer periods (Jakob et al., 1999) via chlororespiratory activity. On the other hand, it was shown by Lepetit et al. (2013) that plastidic redox manipulation can alter the gene expression pattern similarly as in green algae. However, many other studies gave evidence that metabolomic flux regulations in diatoms are different from those of green algae (Wilhelm et al., 2006). For instance, the thioredoxin system in diatoms is only weakly involved in light/dark regulation of the Calvin cycle enzymes (Kroth et al., 2008) and the metabolite exchange between the chloroplast and the cytosol also shows specific peculiarities (Chu et al., 2016). These differences between diatoms and green algae regarding the light acclimation raised the question, whether there are additional sensors besides light-triggered redox state oscillations, which inform the diatom cell about the incident light intensity.

In the aquatic environment of natural habitats, red light is quickly attenuated in the upper water layers, whereas blue light or green light can penetrate much deeper. Therefore, it is likely to assume that the ratio of blue to red light would be a sound indicator for the vertical position in the water body. This would claim for the interaction of a blue light and a red light sensor. Interestingly, diatoms in contrast to green algae possess different phytochromes, which are found to be expressed in physiologically active cells (De Riso et al., 2009). However, their function is still unclear, especially considering that the absorption range of the far-red band of diatom phytochromes is redshifted to wavelengths, which are strongly absorbed by water. It thus was hypothesized that phytochromes in diatoms may receive their excitation light not from the environment, but instead from internal chlorophyll fluorescence (Fortunato et al., 2016). However, an interaction mechanism between blue and red light sensing signalling pathways has not been shown yet.

\subsection{Comparison of BL and RL acclimated wild type and Aureo1a down- regulated cells}

To understand in more detail the specific function of blue and red light for the acclimation reaction in diatoms, Schellenberger Costa et al. (2013) used a special setup to differentiate between light colour signals and light intensity. They cultivated under steady state conditions (chemostat) P. tricornutum cells in BL (469 nm), which is the absorption band of aureochromes and of FCPs, and at $659 \mathrm{~nm}$ (RL), at which most of the quanta are absorbed by the FCPs. They adjusted the quantum flux in a way that RL and BL cells absorbed the same amount of quanta per cell and time under light limiting and light (nearly) saturating conditions. Although the photosynthetic electron flow rate was identical between BL and RL, they observed that the physiological state of the cells was clearly different. Even under low BL, the cells showed a high photoprotective capacity, although an efficient NPQ formation (nonphotochemical quenching) usually is not required under culture conditions. In contrast, cells grown under high RL were not able to acclimate to HL, and could not establish a high NPQ. As a consequence of this blocked light acclimation ability, the cells grown at high RL fluxes showed much lower photon efficiencies than BL cells. Schellenberger Costa et al. (2013) thus concluded that BL is essential for HL acclimation in diatoms. The link of light acclimation to aureochromes became obvious, when an aureochrome1a silencing mutant of $P$. tricornutum cells was subjected to the same experimental conditions. The mutants were not impaired in HL acclimation under BL (instead were acclimating more strongly), but in contrast to wild type cells they could adapt to high RL (Schellenberger Costa et al., 2013). Obviously, the BLsensor AUREO1a can prevent high light acclimation under high RL. This led to the idea, that also in diatoms a blue light receptor might be involved in the cellular response to RL via interaction with phytochrome as shown in land plants (Hughes et al., 2012). In contrast to cryptochromes, aureochromes cannot shift their absorption to red or green light upon excitation regulation. Since aureochromes can act as transcription factors independent on light (see below) a clear explanation for the RL response in diatoms is still lacking.

The results described above led to a second question: How can the down-regulation of a single gene change the phenotype of the cell in a way that requires the coordinated action of many other genes? An answer to this question could be that AUREO1a may act as a transcription factor that influences the expression of many other genes. Indeed, when Schellenberger Costa et al. (2012) analysed the thylakoid proteome of BL and RL acclimated wild type cells, they could show that the amount of a set of proteins increased in BL conditions, which is involved in HL acclimation. Examples are the LHCx1 proteins, necessary for the formation of NPQ, and components of the cytochrome $b_{6} f$ complex, which are increased to enhance the linear electron flow under saturating light intensities. Remarkably, almost all genes encoding those proteins that are involved in light acclimation contain the putative aureochrome-binding motiv "TACGTCA" within in the respective promoter regions.

\subsection{Light quality shift experiments from wild-type and aureochrome1a impaired mutants}

The results reported above indicate that the action of aureochrome (s) may lead to a specific physiological state, which adapts the cell to an optimal performance in different light climates. However, the mechanistic explanation, as of how these cellular phenotypes are generated remains completely enigmatic. Deeper insight in the molecular mechanism(s) of aureochrome-induced physiological acclimation can be obtained by shift experiments, in which RL acclimated cells are transferred to BL and vice versa under identical photon fluxes per cell. Such experiments have been performed by Jungandreas et al. (2014), studying wild type cells as well as aureochrome1a down-regulated mutants. The results can be summarized as follows: A shift from RL to BL is kinetically different from BL to RL and the back reaction from RL to BL is metabolically completely different (unpublished results). Surprisingly, RL-acclimated cells transferred to BL do show a nearly unchanged photosynthetic electron transfer rate, but cell division is completely blocked for about one day. BL acclimated cells shifted to RL instead continue to grow without a change. The analysis of macromolecular composition showed that, during the shifts, the carbon flux allocation is immediately altered: RL-acclimated cells transferred to BL use the assimilated carbon exclusively for protein biosynthesis, whereas BL acclimated cells shifted to RL funnel the carbon solely to carbohydrates. This C-allocation pattern matches perfectly with the short term changes in the metabolome. In contrast, such drastic reactions during shift experiments have not been observed in an aureochromela downregulated mutant (Jungandreas et al., 2014). Therefore, a comparative transcriptome analysis of wild type and aureochrome1a knock-out cells may deliver more detailed information about the aureochrome1a-controlled genes and the regulatory network, for a deeper understanding of the molecular basis of the BL and RL acclimation reactions in diatoms. This work is now in progress.

\subsection{Phenotypes of aureochrome1a and aureochrome1b knock-out mutants of Phaeodactylum tricornutum}

Since in AUREO1a-silenced strains remnant AUREO1a protein could be present, or might be functionally replaced by one of the other aureochromes, AUREO1a and AUREO1b knock-out mutants of P. tricornutum have been generated by genome editing via TALEN technology (Serif et al., 2017). The results of this study has been published by Mann et al. (2017), showing for the first time that AUREO1a-deficient mutants are strongly impaired in acclimation to high BL, as documented by a strong reduction in both photosynthetic and NPQ capacity. In contrast, in RL the differences between the AUREO1a 
deficient mutant and the wild type cells is smaller, but significant. In addition, the phenotype of AUREO1b knock-out mutants is clearly distinguishable from AUREO1a-deficient cells by their ability to establish a high NPQ similar to the wild type in blue light. From this study, it can be concluded that both, AUREO1a and AUREO1b, act as light-acclimating elements but are functionally not identical.

\section{Conclusions}

Aureochromes represent a new type of photoreceptor with so far mostly unknown functions. Although $P$. tricornutum possesses four active aureochromes genes, the respective proteins apparently are not functionally redundant. AUREO1a is a strong regulator in light acclimation and apparently coordinates a concerted action of the reorganisation of different processes: Firstly, components of the thylakoid membrane and the Calvin cycle capacity are affected, which becomes obvious by changes in the maximal photosynthetic activities. Secondly, changes in the enzymatic activity, leading to alterations in the allocation of the assimilated carbon to the major macromolecular pools, coincide with aureochrome activity, but it is not clear yet whether this is due to a direct regulation process or whether it is the result of secondary effects. Finally, aureochromes are involved in regulation of cellular key processes like cell cycle and cell division.

\section{Acknowledgements}

This work was supported by the German Research Foundation (DFG, Germany) within the Research Group FOR1261 (Grants Kr1661/8-1 and 8-2 to PGK, grants Wi764/8-1 and 8-2 to CW, and grants Ko3580/ 1-1 and 1-2 to TK), and a Heisenberg fellowship to TK (Ko3580/4-1), as well as by the University of Konstanz (to PGK).

\section{References}

Akiyama, Y., Nakasone, Y., Nakatani, Y., Hisatomi, O., Terazima, M., 2016. Time-resolved detection of light-induced dimerization of monomeric aureochrome- 1 and change in affinity for DNA. J. Phys. Chem. B 120, 7360-7370.

Archibald John, John M., 2015. Endosymbiosis and eukaryotic cell evolution. Curr. Biol. 25, R911-R921.

Banerjee, A., Herman, E., Kottke, T., Essen, L.-O., 2016a. Structure of a native-like aureochrome 1a LOV domain dimer from Phaeodactylum tricornutum. Structure 24, $171-178$.

Banerjee, A., Herman, E., Serif, M., Maestre-Reyna, M., Hepp, S., Pokorny, R., Kroth, P.G., Essen, L.O., Kottke, T., 2016b. Allosteric communication between DNA-binding and light-responsive domains of diatom class I aureochromes. Nucleic Acids Res. 44, 5957-5970.

Blatt, M.R., Briggs, W.R., 1980. Blue-ligth-induced cortical fiber reticulation concomitant with chloroplast aggregation in the alga Vaucheria sessilis. Planta 147, 355-362.

Bowler, C., Allen, A.E., Badger, J.H., Grimwood, J., Jabbari, K., Kuo, A., Maheswari, A., Martens, C., Maumus, F., Otillar, R.P., Rayko, E., Salamov, A., Vandepoele, K., Beszteri, B., Gruber, A., Heijde, M., Katinka, M., Mock, T., Valentin, K., Vérret, F., Berges, J.A., Brownlee, C., Cadoret, J.P., Chiovitti, A., Choi, C.J., Coesel, S., De Martino, A., Detter, J.D., Durkin, C., Falciatore, A., Fournet, J., Haruta, M., Huysman, M., Jenkins, B.D., Jiroutova, K., Jorgensen, R.E., Joubert, Y., Kaplan, A., Kroeger, N., Kroth, P.G., La Roche, J., Lindquist, E., Lommer, M., Martin-Jézéquel, V., Lopez, P.J., Lucas, S., Mangogna, M., McGinnis, K., Medlin, L.K., Montsant, A., Oudot-Le Secq, M.P., Napoli, N., Obornik, M., Petit, J.L., Porcel, B.L., Poulsen, N., Robison, M., Rychlewski, L., Rynearson, T.A., Schmutz, J., Schnitzler Parker, M., Shapiro, H. Siaut, M., Stanley, M., Sussman, M.R., Taylor, A., Vardi, A., von Dassow, P., Vyverman, W., Willis, A., Wyrwicz, L.S., Rokhsar, D.S., Weissenbach, J., Armbrust, E.V., Green, B.R., Van de Peer, Y., Grigoriev, I.V., 2008. The Phaeodactylum genome reveals the evolutionary history of diatom genomes. Nature 456, 239-244.

Chaves, I., Pokorny, R., Byrdin, M., Hoang, N., Ritz, T., Brettel, K., Essen, L.O., van der Horst, G.T., Batschauer, A., Ahmad, M., 2011. The cryptochromes: blue light photoreceptors in plants and animals. Annu. Rev. Plant Biol. 62, 335-364.

Chen, M., Chory, J., 2011. Phytochrome signaling mechanisms and the control of plant development. Trends Cell Biol. 21, 664-671.

Christie, J.M., 2007. Phototropin blue-light receptors. Annu. Rev. Plant Biol. 58, 21-45.

Chu, L., Gruber, A., Ast, M., Schmitz-Esser, S., Altensell, J., Neuhaus, E., Kroth, P.G., Haferkamp, I., 2016. Shuttling of (deoxy-) purine nucleotides between compartments of the diatom Phaeodactylum tricornutum. New Phytol. 213, 193-205.

Crosson, S., Rajagopal, S., Moffat, K., 2003. The LOV domain family: photoresponsive signaling modules coupled to diverse output domains. Biochemistry 42, 2-10.

De Riso, V., Raniello, R., Maumus, F., Rogato, A., Bowler, C., Falciatore, A., 2009. Gene silencing in the marine diatom Phaeodactylum tricornutum. Nucleic Acids Res. 37, e96.
Deng, Y., Yao, J., Fu, G., Guo, H., Duan, D., 2014. Isolation, Expression and characterization of blue light receptor AUREOCHROME gene from Saccharina japonica (Laminariales, phaeophyceae). Mar. Biotechnol. 16, 135-143.

Depauw, F.A., Rogato, A., Ribera, d.A.M., Falciatore, A., 2012. Exploring the molecular basis of responses to light in marine diatoms. J. Exp. Bot. 63, 1575-1591.

Di Roberto, R.B., Peisajovich, S.G., 2014. The role of domain shuffling in the evolution of signaling networks. J. Exp. Zool. Part B: Mol. Dev. Evol. 322, 65-72.

Duanmu, D., Rockwell, N.C., Clark Lagarias, J., 2017. Algal light sensing and photoacclimation in aquatic environments. Plant Cell Environ. http://dx.doi.org/10.1111/ pce. 12943.

Ernst, O.P., Lodowski, D.T., Elstner, M., Hegemann, P., Brown, L.S., Kandori, H., 2014. Microbial and animal rhodopsins: structures functions, and molecular mechanisms. Chem. Rev. 114, 126-163.

Fortunato, A.E., Jaubert, M., Enomoto, G., Bouly, J.-P., Raniello, R., Thaler, M., Malviya, S., Bernardes, J.S., Rappaport, F., Gentili, B., Carbone, A., Bowler, C., Ribera d'Alcalà, M., Ikeuchi, M., Falciatore, A., 2016. Diatom phytochromes reveal the existence of far-red light based sensing in the ocean. Plant Cell 28, 616-628.

Foster, K.W., Saranak, J., Patel, N., Zarilli, G., Okabe, M., Kline, T., Nakanishi, K., 1984. A rhodopsin is the functional photoreceptor for phototaxis in the unicellular eukaryote Chlamydomonas. Nature 311, 756-759.

Gabryś, H., Walczak, T., Haupt, W., 1984. Blue-light-induced chloroplast orientation in Mougeotia. Evidence for a separate sensor pigment besides phytochrome. Planta 160, $21-24$.

Glantz, S.T., Carpenter, E.J., Melkonian, M., Gardner, K.H., Boyden, E.S., Wong, G.K.-S., Chow, B.Y., 2016. Functional and topological diversity of LOV domain photoreceptors. Proc. Natl. Acad. Sci. U. S. A. 113, 1442-1451.

Halldal, P., 1958. Action spectra of phototaxis and relation problem in volvocalcs, ulvagamete and dinophyceae. Physiol. Plant. 11, 118-153.

Harper, S.M., Neil, L.C., Gardner, K.H., 2003. Structural basis of a phototropin light switch. Science 301, 1541-1544.

Harper, S.M., Christie, J.M., Gardner, K.H., 2004. Disruption of the LOV-Ja helix interaction activates phototropin kinase activity. Biochemistry 43, 16184-16192.

Hegemann, P., 2008. Algal sensory photoreceptors. Annu. Rev. Plant Biol. 59, 167-189.

Heintz, U., Schlichting, I., 2016. Blue light-induced LOV domain dimerization enhances the affinity of Aureochrome 1a for its target DNA sequence. eLife 5, e11860.

Herman, E., Kottke, T., 2015. Allosterically regulated unfolding of the A'a helix exposes the dimerization site of the blue-light-sensing aureochrome-LOV domain. Biochemistry 54, 1484-1492.

Herman, E., Sachse, M., Kroth, P.G., Kottke, T., 2013. Blue-light-induced unfolding of the $\mathrm{J} a$ helix allows for the dimerization of aureochrome-LOV from the diatom Phaeodactylum tricornutum. Biochemistry 52, 3094-3101.

Herrou, J., Crosson, S., 2011. Function, structure and mechanism of bacterial photosensory LOV proteins. Nat. Rev. Micro 9, 713-723.

Hisatomi, O., Nakatani, Y., Takeuchi, K., Takahashi, F., Kataoka, H., 2014. Blue lightinduced dimerization of monomeric aureochrome-1 enhances its affinity for the target sequence. J. Biol. Chem. 289, 17379-17391.

Hughes, R.M., Vrana, J.D., Song, J., Tucker, C.L., 2012. Light-dependent, dark-promoted interaction between Arabidopsis cryptochrome 1 and phytochrome B proteins. J. Biol. Chem. 287, 22165-22172.

Huysman, M.J., Fortunato, A.E., Matthijs, M., Costa, B.S., Vanderhaeghen, R., Van den Daele, H., Sachse, M., Inze, D., Bowler, C., Kroth, P.G., Wilhelm, C., Falciatore, A., Vyverman, W., De, V.L., 2013. AUREOCHROME1a-mediated induction of the diatomspecific cyclin dsCYC2 controls the onset of cell division in diatoms (Phaeodactylum tricornutum). Plant Cell 25, 215-228.

Ishikawa, M., Takahashi, F., Nozaki, H., Nagasato, C., Motomura, T., Kataoka, H., 2009. Distribution and phylogeny of the blue light receptors aureochromes in eukaryotes. Planta 230, 543-552.

Ishikawa, M., Kataoka, H., Takahashi, F., 2012. Analysis of Light-dependent cell morphology and an accumulation response in Ochromonas danica. Cytologia (Tokyo) 77, 465-473.

Jakob, T., Goss, R., Wilhelm, C., 1999. Activation of diadinoxanthin de-epoxidase due to a chlororespiratory proton gradient in the dark in the diatom Phaeodactylum tricornutum. Plant Biol. 1, 76-82.

Jia, N., Lia, L., Lina, L., Lina, S., 2017. Identification and expression analysis of blue light receptor aureochrome in the harmful alga Heterosigma akashiwo (Raphidophyceae). Harmful Algae 61, 71-79.

Jungandreas, A., Schellenberger Costa, B., Jakob, T., von Bergen, M., Baumann, S., Wilhelm, C., 2014. The acclimation of Phaeodactylum tricornutum to blue and red light does not influence the photosynthetic light reaction but strongly disturbs the carbon allocation pattern. PLoS One 9, e99727.

König, S., Juhas, M., Jäger, S., Kottke, T., Büchel, C., 2017. The cryptochrome - photolyase protein family in diatoms. J. Plant Physiol. 217, 15-19.

Kataoka, H., 1979. Phototropic responses of Vaucheria geminata to intermittent blue light stimuli. Plant Physiol. 63, 1107-1110.

Kennis, J.T.M., Mathes, T., 2013. Molecular eyes: proteins that transform light into biological information. Interface Focus 3, 20130005.

Kerruth, S., Ataka, K., Frey, D., Schlichting, I., Heberle, J., 2014. Aureochrome 1 illuminated: structural changes of a transcription factor probed by molecular spectroscopy. PLoS One 9, e103307.

Kianianmomeni, A., Hallmann, A., 2014. Algal photoreceptors: in vivo functions and potential applications. Planta 239, 1-26.

Kottke, T., Oldemeyer, S., Wenzel, S., Zou, Y., Mittag, M., 2017. Cryptochrome photoreceptors in green algae: unexpected versatility of mechanisms and functions. J. Plant Physiol. 217, 4-14.

Kroth, P.G., Chiovitti, A., Gruber, A., Martin-Jezequel, V., Mock, T., Parker, M.S., Stanley, M.S., Kaplan, A., Caron, L., Weber, T., Maheswari, U., Armbrust, E.V., Bowler, C., 
2008. A model for carbohydrate metabolism in the diatom Phaeodactylum tricornutum deduced from comparative whole genome analysis. PLoS One 3, E1426.

Kroth, P.G., 2015. The biodiversity of carbon assimilation. J. Plant Physiol. 172, 76-81.

Lepetit, B., Volke, D., Szabo, M., Hoffmann, R., Garab, G., Wilhelm, C., Goss, R., 2007. Spectroscopic and molecular characterization of the oligomeric antenna of the diatom Phaeodactylum tricornutum. Biochemistry 46, 9813-9822.

Lepetit, B., Sturm, S., Rogato, A., Gruber, A., Sachse, M., Falciatore, A., Kroth, P.G., Lavaud, J., 2013. High light acclimation in the secondary plastids containing diatom Phaeodactylum tricornutum is triggered by the redox state of the plastoquinone pool. Plant Physiol. 161, 853-865.

Li, F.-W., Villarreal, J.C., Kelly, S., Rothfels, C.J., Melkonian, M., Frangedakis, E., Ruhsam, M., Sigel, E.M., Der, J.P., Pittermann, J., Burge, D.O., Pokorny, L., Larsson, A., Chen, T., Weststrand, S., Thomas, P., Carpenter, E., Zhang, Y., Tian, Z., Chen, L., Yan, Z., Zhu, Y., Sun, X., Wang, J., Stevenson, D.W., Crandall-Stotler, B.J., Shaw, A.J., Deyholos, M.K., Soltis, D.E., Graham, S.W., Windham, M.D., Langdale, J.A., Wong, G.K.-S., Mathews, S., Pryer, K.M., 2014. Horizontal transfer of an adaptive chimeric photoreceptor from bryophytes to ferns. Proc. Natl. Acad. Sci. U. S. A. 111, 6672-6677.

Li, F.-W., Rothfels, C.J., Melkonian, M., Villarreal, J.C., Stevenson, D.W., Graham, S.W., Wong, G.K.S., Mathews, S., Pryer, K.M., 2015. The origin and evolution of phototropins. Front. Plant Sci. 6, 637.

Losi, A., Gärtner, W., 2008. Shedding (blue) light on algal gene expression. Proc. Natl. Acad. Sci. U. S. A. 105, 7-8.

Losi, A., 2004. The bacterial counterparts of plant phototropins. Photochem. Photobiol, Sci. 3, 566-574.

Mann, M., Serif, M., Jakob, T., Kroth, P.G., Wilhelm, C., 2017. PtAUREO1a and PtAUREO1b knockout mutants of the diatom Phaeodactylum tricornutum are blocked in photoacclimation to blue light. J. Plant Physiol. 217, 44-48. http://dx.doi. org/10.1016/j.jplph.2017.05.020.

Matsuoka, D., Tokutomi, S., 2005. Blue light-regulated molecular switch of Ser/Thr kinase in phototropin. Proc. Natl. Acad. Sci. U. S. A. 102, 13337-13342.

Mitra, D., Yang, X., Moffat, K., 2012. Crystal structures of aureochrome1 LOV suggest new design strategies for optogenetics. Structure 20, 698-706.

Mittag, M., 2017. Light driven reactions in model algae. J. Plant Physiol 217, 1-3.

Nash, A.I., McNulty, R., Shillito, M.E., Swartz, T.E., Bogomolni, R.A., Luecke, H., Gardner, K.H., 2011. Structural basis of photosensitivity in a bacterial light-oxygen-voltage/ helix-turn-helix (LOV-HTH) DNA-binding protein. Proc. Natl. Acad. Sci. U. S. A. 108, 9449-9454.

Rockwell, N.C., Duanmu, D., Martin, S.S., Bachy, C., Price, D.C., Bhattacharya, D., Worden, A.Z., Lagarias, J.C., 2014. Eukaryotic algal phytochromes span the visible spectrum. Proc. Natl. Acad. Sci. U. S. A. 111, 3871-3876.

Schellenberger Costa, B., Jungandreas, A., Jakob, T., Weisheit, W., Mittag, M., Wilhelm, C., 2012. Blue light is essential for high light acclimation and photoprotection in the diatom Phaeodactylum tricornutum. J. Exp. Bot. 64, 483-493.

Schellenberger Costa, B., Sachse, M., Jungandreas, A., Bartulos, C.R., Gruber, A., Jakob,
T., Kroth, P.G., Wilhelm, C., 2013. Aureochrome 1a is involved in the photoacclimation of the diatom Phaeodactylum tricornutum. PLoS One 8, e74451.

Serif, M., Lepetit, B., Weißert, K., Kroth, P.G., Rio Bartulos, C., 2017. A fast and reliable strategy to generate TALEN-mediated gene knockouts in the diatom Phaeodactylum tricornutum. Algal Res. 23, 186-195.

Shihira-Ishikawa, I., Nakamura, T., Higashi, S., Watanabe, M., 2007. Distinct responses of chloroplasts to blue and green laser microbeam irradiations in the centric diatom Pleurosira laevis. Photochem. Photobiol. 83, 1101-1109.

Suetsugu, N., Mittmann, F., Wagner, G., Hughes, J., Wada, M., 2005. A chimeric photoreceptor gene, NEOCHROME, has arisen twice during plant evolution. Proc. Natl. Acad. Sci. U. S. A. 102, 13705-13709.

Suzuki, T., Yamasaki, K., Fujita, S., Oda, K., Iseki, M., Yoshida, K., Watanabe, M., Daiyasu, H., Toh, H., Asamizu, E., Tabata, S., Miura, K., Fukuzawa, H., Nakamura, S., Takahashi, T., 2003. Archaeal-type rhodopsins in Chlamydomonas: model structure and intracellular localization. Biochem. Biophys. Res. Commun. 301, 711-717.

Swartz, T.E., Corchnoy, S.B., Christie, J.M., Lewis, J.W., Szundi, I., Briggs, W.R. Bogomolni, R.A., 2001. The photocycle of a flavin-binding domain of the blue light photoreceptor phototropin. J. Biol. Chem. 276, 36493-36500.

Takahashi, F., Hishinuma, T., Kataoka, H., 2001. Blue light-induced branching in vaucheria: requirement of nuclear accumulation in the irradiated region. Plant Cell Physiol. 42, 274-285.

Takahashi, F., Yamagata, D., Ishikawa, M., Fukamatsu, Y., Ogura, Y., Kasahara, M., Kiyosue, T., Kikuyama, M., Wada, M., Kataoka, H., 2007. AUREOCHROME: a photoreceptor required for photomorphogenesis in stramenopiles. Proc. Natl. Acad. Sci. U. S. A. 104, 19625-19630.

Tikkanen, M., Gollan, P.J., Suorsa, M., Kangasjarvi, S., Aro, E.M., 2012. STN7 operates in retrograde signaling through controlling redox balance in the electron transfer chain. Front. Plant Sci. 3, 277.

Toyooka, T., Hisatomi, O., Takahashi, F., Kataoka, H., Terazima, M., 2011. Photoreactions of aureochrome-1. Biophys. J. 100, 2801-2809.

Vieler, A., Wu, G., Tsai, C.-H., Bullard, B., Cornish, A.J., Harvey, C., Reca, I.-B., Thornburg, C., Achawanantakun, R., Buehl, C.J., Campbell, M.S., Cavalier, D., Childs, K.L., Clark, T.J., Deshpande, R., Erickson, E., Ferguson, A.A., Handee, W., Kong, Q., Li, X., Liu, B., Lundback, S., Peng, C., Roston, R.L., Sanjaya Simpson, J.P., TerBush, A., Warakanont, J., Zäuner, S., Farre, E.M., Hegg, E.L., Jiang, N., Kuo, M.-H., Lu, Y., Niyogi, K.K., Ohlrogge, J., Osteryoung, K.W., Shachar-Hill, Y., Sears, B.B., Sun, Y., Takahashi, H., Yandell, M., Shiu, S.-H., Benning, C., 2012. Genome, Functional gene annotation and nuclear transformation of the heterokont oleaginous alga Nannochloropsis oceanica CCMP1779. PLoS Genet. 8, e1003064.

Wilhelm, C., Büchel, C., Fisahn, J., Goss, R., Jakob, T., LaRoche, J., Lavaud, J., Lohr, M., Riebesell, U., Stehfest, K., Valentin, K., Kroth, P.G., 2006. The regulation of carbon and nutrient assimilation in diatoms is significantly different from green algae. Protist 157, 91-124.

Wilhelm, C., Jungandreas, A., Jakob, T., Goss, R., 2014. Light acclimation in diatoms: from phenomenology to mechanisms. Mar. Genomics 16, 5-15. 